eISSN 2444-7986

DOI: https://doi.org/10.14201/orl201674.14370

\author{
Artículo original
}

\title{
CORDECTOMÍA POSTERIOR EN EL TRATAMIENTO DE LA PARÁLISIS BILATERAL DE CUERDAS VOCALES. ¿DEBE SER LA EDAD UNA LIMITACIÓN EN SU INDICACIÓN?
}

\section{Posterior cordectomy in the treatment of bilateral paralysis of the vocal cords. Should be the age a restriction in your surgery?}

\author{
Julio RAMA-LÓPEZ; Claudio CARNEVALE; Manuel TOMÁS-BARBERÁN \\ Servicio ORL. Hospital Universitario Son Espases. Palma de Mallorca. Islas Baleares. España. \\ Correspondencia: julioramalopez@hotmail.com
}

Fecha de recepción: 25 de abril de 2016

Fecha de aceptación: 13 de junio de 2016

Fecha de Publicación: 17 de junio de 2016

Conflicto de intereses: Los autores declaran no tener conflictos de intereses

Imágenes: Los autores declaran haber obtenido las imágenes con el permiso de los pacientes

Política de derechos y autoarchivo: se permite el autoarchivo de la versión post-print (SHERPA/RoMEO)

Licencia CC BY-NC-ND. Licencia Creative Commons Atribución-NoComercial-SinDerivar 4.0 Internacional

(C) Universidad de Salamanca. Su comercialización está sujeta al permiso del editor

RESUMEN

Introducción y objetivo: La parálisis bilateral de cuerdas vocales es rara pero potencialmente letal. El objetivo de este estudio es evaluar los resultados obtenidos con la técnica de cordectomía posterior microscópica con láser $\mathrm{CO}_{2}$ y valorar si existe un mayor riesgo de complicaciones en los pacientes mayores de 70 años. Material y método: 18 pacientes divididos en grupo A (menores de 70 años; 10 pacientes) y grupo B (mayores de 70 años; 8 pacientes). Resultados: Se obtuvo un buen resultado respiratorio en el $94 \%$ de los pacientes con una tasa de revisión del $6 \%$. La incidencia de voz áfona y necesidad de rehabilitación de la deglución fue mayor en el grupo B. Conclusiones: La cordectomía posterior con láser es un tratamiento adecuado en la parálisis laríngea bilateral. Los pacientes mayores de 70 años deben ser valorados específicamente al presentar mayor riesgo de aspiración.

PALABRAS CLAVE laringe; nervio recurrente; parálisis laríngea; láser; cordectomía

SUMMARY Introduction and objective: Bilateral vocal cord paralysis is a rare but potentially lethal condi-
tion. The objective of this retrospective study is to evaluate the results obtained with the
technique of endoscopic $\mathrm{CO}_{2}$ laser posterior cordectomy and comparatively assess whether
there is a higher risk of complications in patients older than 70 years. Methods: 18 consecu-
tive patients classified in group A (under 70 years, 10 patients) and group B (over 70 years, 8
patients). Results: Good respiratory outcome in $94 \%$ of patients with a revision rate of $6 \%$
was obtained. The incidence of dysphonia and rehabilitation swallowing maneuvers was
higher in the group B. Conclusions: Posterior cordectomy is a good treatment in bilateral
laryngeal paralysis patients. We have to keep in mind those patients over 70 years must be
evaluated in a specific way because they have a greater risk of aspiration.

KEYWORDS larynx; recurrent nerve; laryngeal paralysis; laser cordectomy 


\section{INTRODUCCIÓN}

La parálisis bilateral de las cuerdas vocales representa una condición rara pero potencialmente letal que a menudo requiere una intervención quirúrgica de urgencia. Los síntomas más frecuentes son roncopatía, apneas del sueño, disnea en varios grados de intensidad e incluso la muerte en pacientes con reserva pulmonar reducida. Puede presentarse como cuadro de disnea brusco (en el postoperatorio inmediato) pero lo más frecuente es que se presente como disnea de pequeños o moderados esfuerzos. No suele causar disfonía ni problemas deglutorios [1, 2].

El origen de la parálisis puede encontrarse en el tórax, en el cuello y en el cráneo. Sin duda la causa más común de parálisis bilateral de las cuerdas vocales es la lesión periférica del nervio recurrente durante la cirugía tiroidea (26-59\%). Otras causas iatrogénicas son la cirugía torácica y cervical y el traumatismo por intubación (1-31\%), neoplasias cervicotorácicas $(5-17 \%)$, traumatismos que afectan la región de cabeza y cuello, causas idiopáticas, neurológicas, neuromusculares e inflamatorias constituyen otros factores etiológicos de interés (7-22\%) [1].

El objetivo del tratamiento es obtener un adecuado paso aéreo y al mismo tiempo preservar el habla y la deglución hasta alcanzar el mejor equilibrio entre el resultado respiratorio y fonatorio sin aspiración.

Hasta los primeros años del siglo XX, la traqueotomía fue el único tratamiento de la parálisis bilateral de las cuerdas vocales.

Actualmente a pesar de seguir siendo un tratamiento eficaz, sobre todo en emergencia, no es un procedimiento deseable a largo plazo. Por ello, han sido descritas técnicas quirúrgicas diferentes con el objetivo de asegurar una vía aérea aceptable evitando la traqueotomía y sin afectar el resto de funciones laríngeas.

En 1922, Chevalier Jackson describió la cordoventriculectomía, o sea la resección unilateral de la cuerda vocal y la banda ventricular ipsilateral, con mejoría en la respiración y con la posibilidad de decanular algunos pacientes; el inconveniente era una pérdida evidente de fuerza de la voz. Diez años más tarde, en 1932, Hoover propuso la resección submucosa de las cuerdas vocales, pero este procedimiento causaba generalmente fibrosis por cicatrización y estenosis glótica con disnea severa. En 1939, King -cirujano ortopédico-, introdujo una nueva técnica que consistía en suturar el cartílago aritenoides al músculo omohioideo (aritenoidopexia). En 1946 Woodman describió un procedimiento que fue popular durante los años 50: a través de un abordaje extralaríngeo realizaba una aritenoidectomía y suturaba el proceso vocal al cuerno inferior del cartílago tiroides para lateralizar la cuerda vocal. El abordaje endolaríngeo fue introducido por primera vez por Thornell en 1949 cuando realizó una aritenoidectomía endoscópica con electrocauterio con una tasa de éxito del $80 \%$. Eskew y Bailey fueron los primeros en usar el láser $\mathrm{CO} 2$ para realizar una aritenoidectomía en un modelo animal en 1983 y esta técnica fue adaptada para pacientes con parálisis cordal bilateral por Ossoff en 1984. En 1989 Dennis y Kashima introdujeron la cordectomía posterior endoscópica con láser $\mathrm{CO}_{2}$ que consiste en la escisión de la porción posterior de la cuerda vocal sin aritenoidectomía. En 1994 Eckel et al. compararon la aritenoidectomía láser con la cordectomía posterior láser y observaron que ambas técnicas tenían una tasa de éxito superior al $90 \%$ pero la última era más fácil de realizar y presentaba una tasa inferior de aspiración subclínica [2, 3].

Las técnicas quirúrgicas utilizadas para el tratamiento de la parálisis bilateral de las cuerdas vocales se clasifican en dos grupos: resectivas y no resectivas. Entre las primeras, que son las más utilizadas, está la cordectomía (el mayor inconveniente es la disfonía), la aritenoidectomía (que produce aspiración y necrosis cricoidea en pacientes irradiados, aunque se han desarrollado técnicas que reducen este riesgo) y la cordectomía posterior. Entre los procedimientos no resectivos tenemos la aritenoidopexia (potencialmente reversible, pero con resultados que empeoran en el tiempo), la reinervación laríngea (descrita por Tucker en 1976) [4] —técnicamente difícil y que ha ido perdiendo importancia- y la colocación de dispositivos que detectan la funcionalidad laríngea actuando como marcapasos -técnicamente difícil y con una menor difusión por su complejidad- [5].

Con la exclusión de la técnica de reinervación, en todas las intervenciones quirúrgicas se sacrifica voz por la respiración.

Sin embargo, la existencia de una laringe fija a la que debemos ampliar el hiato respiratorio introduce la consideración de si en el contexto 
de una cirugía convertiremos con algunas técnicas el problema respiratorio en un problema de aspiración.

Este aspecto, cobraría especial transcendencia en pacientes de edad avanzada que ya de manera silente podrían padecer aspiraciones y cuya evolución postoperatoria sería de mayor riesgo ante una posible aspiración [6-8].

Así, el objetivo de este estudio es evaluar los resultados obtenidos con la técnica de cordectomía posterior endoscópica con láser $\mathrm{CO}_{2}$ en nuestra serie de 18 pacientes y valorar de manera comparativa si existe un mayor riesgo de complicaciones en los pacientes mayores de 70 años. De esta manera pretendemos establecer si la edad debe suponer una modificación en la indicación de esta técnica que consideramos de elección en el tratamiento de la parálisis laríngea bilateral.

\section{MATERIAL Y MÉTODO}

Estudio retrospectivo sobre 18 pacientes diagnosticados de parálisis cordal bilateral sometidos a cordectomía posterior láser en los últimos 8 años.

Se excluyeron de este estudio los pacientes en edad pediátrica (menores de 14 años), las originadas por causa oncológica o por fijación posterior, que recibieron tratamientos diferentes al descrito en el presente estudio.

La edad media fue de 60,5 años (rango 2286). Para valorar la posible influencia de la edad, se formaron dos grupos de pacientes: menores de 70 años - 10 pacientes (grupo A; edad media 33 años) - y mayores de 70 años -8 pacientes (grupo $\mathrm{B}$; edad media 77,75 años)_. Se realizó un estudio comparativo entre ambos.

\section{VALORACIÓN PREOPERATORIA}

Los 18 pacientes de la serie fueron diagnosticados de parálisis bilateral en nuestro servicio procediéndose a una recogida exhaustiva de datos, que incluyeron edad, tiempo de evolución, tratamientos previos, sintomatología, etc.

La parálisis bilateral que presentaban los pacientes se produjo, en un tercio de los pacientes ( 6 pacientes) después de la realización de una tiroidectomía total, 3 pacientes después de un traumatismo craneoencefálico, 3 pacientes con antecedente de radioterapia cervical hacía más de 10 años, 2 pacientes con intubaciones prolongadas, 1 paciente con diagnóstico de carcinoma de esófago, 1 pa- ciente con adenopatías mediastínicas, 1 paciente de etiología idiopática y un paciente posthematoma cervical tras endarterectomía (Tabla 1). En todos ellos se realizó una valoración de la función respiratoria preoperatoria y se realizó un TAC cervicotorácico.

En 4 pacientes del grupo $A$ y 4 pacientes del grupo B se había realizado una traqueotomía previa.

Todos los pacientes tenían una parálisis bilateral de más de 6 meses de evolución que les producía una disnea con leves o moderados esfuerzos que limitaba su actividad diaria.

\section{INTERVENCIÓN QUIRÚRGICA}

Los 18 pacientes de la serie fueron intervenidos bajo anestesia general con tubo endotraqueal para láser con laringoscopia directa en suspensión.

Se realizó cordectomía de la mitad posterior de una cuerda vocal bajo control microscópico a distancia focal de $400 \mathrm{~mm}$ con micromanipulador láser AcuBlade $($, según protocolo de laringe a potencia de $10 \mathrm{~W}$ en modo continuo superpulse (Figura 1) [9-16].

Los pacientes fueron extubados en el propio quirófano.

\section{CUIDADOS POSTOPERATORIOS}

Todos los pacientes siguieron tratamiento corticoideo postoperatorio durante una semana en pauta descendente, tratamiento antibiótico con amoxicilina y ácido clavulánico durante una semana y omeprazol a una dosis de 40 $\mathrm{mg} /$ día durante 3 meses.

A las 24 horas de la cirugía fueron valorados en la unidad de disfagia y aspiración de nuestro servicio, que indicaba el inicio de dieta vía oral con maniobras facilitadoras de la deglución en los casos que se consideró necesario. Todos los pacientes no portadores de traqueotomía previa fueron dados de alta a las 48 horas de la cirugía. Los 8 pacientes traqueotomizados previamente se mantuvieron ingresados hasta el cierre del estoma durante 1 semana.

Los 18 pacientes del estudio han seguido controles periódicos en nuestras consultas externas de manera bimensual al menos durante un año (Figura 2).

Los cálculos estadísticos se realizaron con el programa SPSS v.21, utilizándose el análisis de datos descriptivos y los tests para variables paramétricas (t de Student) y no paramétricas (U de Mann-Whitney). 
Tabla 1. Cordectomía posterior en parálisis bilateral de cuerdas vocales. Resumen de la serie de 18 pacientes.

\begin{tabular}{|c|c|c|c|c|c|c|}
\hline & Edad & Etiología & $\begin{array}{c}\text { Traqueotomía } \\
\text { previa }\end{array}$ & Reintervención & Complicaciones & VHI-10 \\
\hline Caso 1 & 70 & Tiroidectomía & + & & & 8 \\
\hline Caso 2 & 51 & Tiroidectomía & + & & & 33 \\
\hline Caso 3 & 82 & Tiroidectomía & & & & 13 \\
\hline Caso 4 & 67 & Tiroidectomía & & + & & 4 \\
\hline Caso 5 & 81 & Tiroidectomía & & & & 21 \\
\hline Caso 6 & 68 & Tiroidectomía & + & & $\begin{array}{l}\text { Traqueotomía } \\
\text { urgente }\end{array}$ & 18 \\
\hline Caso 7 & 84 & Radioterapia cervical & & & & $\mathrm{F}$ \\
\hline Caso 8 & 42 & Radioterapia cervical & & & & 29 \\
\hline Caso 9 & 58 & Radioterapia cervical & & & & 27 \\
\hline Caso 10 & 21 & Intubación prolongada & & & & 24 \\
\hline Caso 11 & 22 & Intubación prolongada & + & & & 6 \\
\hline Caso 12 & 23 & TCE & & & & 17 \\
\hline Caso 13 & 80 & TCE & & & & 8 \\
\hline Caso 14 & 71 & Endarterectomía & + & & & 17 \\
\hline Caso 15 & 84 & Idiopática & + & & $\begin{array}{l}\text { Neumonía } \\
\text { aspiración }\end{array}$ & 11 \\
\hline Caso 16 & 57 & Carcinoma esofágico & & & & $\mathrm{F}$ \\
\hline Caso 17 & 70 & $\begin{array}{l}\text { Traumatismo con lesión } \\
\text { medular }\end{array}$ & + & & & 4 \\
\hline Caso 18 & 60 & Adenopatía mediastínica & + & & & 9 \\
\hline
\end{tabular}

VHI: Voice Handicap Index

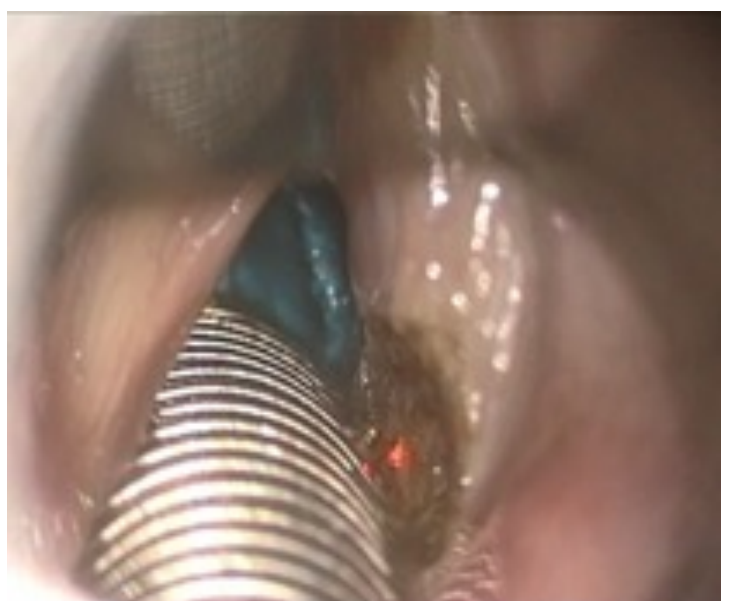

Figura 1. Imagen intraoperatoria de cordectomía posterior de cuerda vocal derecha.

\section{RESULTADOS}

En el $94 \%$ de los pacientes se obtuvo un resultado respiratorio satisfactorio a largo plazo. Los 17 pacientes realizaban sus actividades cotidianas de manera normal experimentando únicamente un paciente joven una limitación leve con el ejercicio físico intenso.

El ingreso medio en el total de la muestra fue de 5,5 días.

En nuestra serie la causa más frecuente de parálisis bilateral de cuerdas vocales fue la tiroidectomía total $(33,3 \%)$, seguida del ante-

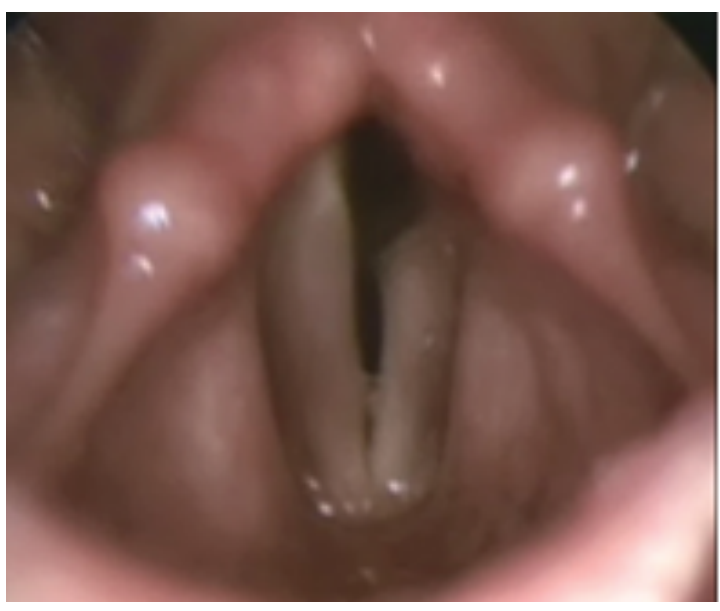

Figura 2. Resultado postoperatorio a los 3 meses de cordectomía posterior izquierda.

cedente de radioterapia cervical y el traumatismo craneoencefálico $(16,7 \%)$.

Tres de los pacientes describían su voz como áfona o falta de fuerza lo que suponía una limitación para mantener conversaciones en ambiente ruidoso y en dos de ellos para hablar por teléfono.

Un paciente mantuvo dificultad respiratoria durante el ingreso por lo que se decidió a los 5 días proceder a su revisión quirúrgica realizándose una cordectomía del tercio posterior de la cuerda vocal contralateral (lo que representa una tasa de revisión del 6\%). Tras esta 
segunda cirugía presentó una gran mejoría recibiendo el alta hospitalaria a las 72 horas.

El paciente con mal resultado presentó a las 48 horas un cuadro de disnea aguda con edema faringolaríngeo por lo que se realizó una traqueotomía de urgencia. No fue posible la decanulación pese a completar una cordectomía bilateral a los 10 días.

En 7 de los 8 pacientes con traqueotomía previa fue posible alcanzar una decanulación con buena tolerancia en el postoperatorio inmediato.

En el análisis comparativo entre grupos, no se encontraron diferencias estadísticamente significativas $(p>0.05)$ en los parámetros preoperatorios, salvo que el grupo B presentaba mayor tasa de morbilidades asociadas (diabetes mellitus, hipertensión arterial y enfermedad pulmonar obstructiva crónica).

No hubo diferencias en la distribución entre grupos de las causas que provocaron la parálisis bilateral $(p>0.05)$.

Dos pacientes presentaron complicaciones postoperatorias. El paciente previamente comentado, con mal resultado, que pertenecía al grupo A y un paciente del grupo $B$ que presentó una neumonía por aspiración que precisó de tratamiento antibiótico endovenoso, lo que prolongó su ingreso hospitalario hasta los 21 días.

Los 3 pacientes que relataban dificultad por falta de intensidad de su voz pertenecían al grupo B (37,5\% de los pacientes), siendo esta diferencia estadísticamente significativa ( $p \leq 0.05)$.

Ningún paciente de la serie presentó disfagia o aspiración en el medio y largo plazo, aunque un número significativamente mayor de pacientes en el grupo B precisaron maniobras facilitadoras de la deglución durante el postoperatorio inmediato ( $\mathrm{s} 0.05)$. Cuatro pacientes del grupo B (50\% de los pacientes) y uno del grupo A (9\%) mantuvieron este tratamiento rehabilitador durante 1 mes.

No se encontraron diferencias estadísticamente significativas en el tiempo de ingreso hospitalario entre ambos grupos ( $p>0.05)$.

Dado el número limitado de pacientes de la serie la potencia estadística debería ser refrendada en estudios con muestras mayores.

\section{DISCUSIÓN}

La parálisis bilateral de cuerdas vocales es una patología que puede poner en riesgo la vida del paciente. Por eso, de manera muy frecuente precisa una intervención quirúrgica para prevenir una dificultad respiratoria extrema. Además, en muchos de los casos, lo que se produce es una limitación en las actividades de la vida diaria que obliga a realizar un procedimiento para mejorar la calidad de vida del paciente. La técnica quirúrgica elegida debe mejorar la vía aérea produciendo un mínimo impacto sobre las funciones deglutorias o de producción de la voz de la laringe y, además, debe intentar evitar la morbilidad asociada a la realización de una traqueotomía permanente.

En nuestro hospital se han abandonado otro tipo de procedimientos optando como técnica de elección por la cordectomía posterior con láser $\mathrm{CO}_{2}$.

Se trata de una técnica sencilla, fácil de realizar y con unos buenos resultados en todos los protocolos revisados. En nuestra serie se obtiene un buen resultado en el $94 \%$ de los pacientes comparable al de otras [9-16].

La elección de esta técnica en lugar de las técnicas que buscan la lateralización de una cuerda vocal se basa en que se obtienen resultados comparables, pero con una menor morbilidad y con un procedimiento más corto y sencillo. Consideramos que otras técnicas de resección como la aritenoidectomía [2, 1820] son más agresivas y mantienen una tasa de complicaciones mayor y otras como la cordotomía pueden no ser suficientemente amplias para obtener un adecuado diámetro glótico en el caso de pacientes con laringes estrechas o necesitar de procedimientos bilaterales lo que puede dificultar la cicatrización o producir un mayor número de eventos aspirativos [3]. Respetar la integridad de los aritenoides y de la comisura posterior así como de las estructuras supraglóticas disminuye la tasa de posibles complicaciones [21, 22].

Otros dispositivos diferentes al láser $\mathrm{CO}_{2}$ se han descrito para la realización de esta técnica $[23,24]$. Sin embargo, todas presentan un índice similar de éxito por lo que el uso del láser carbónico resulta en nuestro caso de elección debido a la amplia experiencia que se dispone en su uso en cirugía oncológica. Proporciona un corte seguro y limpio sin limitar la visibilidad del campo quirúrgico y produce una contracción del tejido remanente que permite una ampliación del espacio aéreo sin necesidad de extraer una mayor cantidad de tejido.

Al tratarse de un procedimiento corto, con pocos riesgos y baja morbilidad asociada, 
puede repetirse en caso de resultados no satisfactorios, procediéndose a realizar un desbridamiento de la intervención previa realizada o completar una cordectomía bilateral algo menos agresiva en la cuerda vocal no intervenida en el primer procedimiento [3, 13, 26]. Esta fue la técnica realizada en uno de los pacientes de nuestra serie obteniéndose un buen resultado final (tasa de revisión del $6 \%$, similar al del resto de series publicadas) [9-17].

En nuestra experiencia, para la realización de esta técnica no es necesaria la realización de una traqueotomía previa. Incluso en nuestro protocolo se ha procedido a la decanulación de 7 de los 8 pacientes a los que se había realizado previamente. Creemos que la realización de una traqueotomía aumentaría la posibilidad de una aspiración en el postoperatorio, retrasaría el inicio de la ingesta oral y aumentaría la estancia hospitalaria sin aumentar la posibilidad de obtener un buen resultado. Quizás esta alta tasa de decanulación se deba a que abordamos de una manera más agresiva la obtención de un adecuado paso aéreo a expensas de una menor calidad vocal.

Aunque se mantiene un buen resultado en cuanto a la voz postoperatoria comparativamente a otras series $[10,14,27]$, es cierto que, en nuestra serie, el $16.6 \%$ de los pacientes relataban alguna dificultad por falta de potencia en su voz. Este grupo de pacientes con un resultado secundario más pobre pertenecían todos ellos al grupo B. Posiblemente cabe mencionar que la edad elevada puede condicionar este resultado al añadirse al defecto producido por la cirugía un componente de presbifonía con menos volumen de la cuerda contralateral [28, 29]. Los 3 pacientes eran portadores de traqueotomía antes de la cirugía por lo que la resección de la cuerda pudo ser quizás algo más agresiva teniendo como objetivo una decanulación temprana. Por tanto, debe tenerse en cuenta que la indicación de una cordectomía posterior en pacientes mayores según nuestros resultados tendrá un porcentaje de disfonía mayor que en el resto de la población.

Creemos de especial trascendencia una valoración temprana de la posible existencia de disfagia o aspiración tras la realización de este tipo de cirugías. Atendiendo a nuestros resultados, esta valoración se hace impres- cindible en pacientes mayores ya que se necesita con una frecuencia significativamente mayor una rehabilitación de la deglución en el postoperatorio inmediato [6-8].

Cabe mencionar que en dos casos de nuestra serie la cordectomía posterior fue realizada en pacientes sometidos a tratamiento oncológico paliativo. En ambos casos se obtuvo un buen resultado falleciendo los pacientes por causas relacionadas con su enfermedad a los 12 y 13 meses sin la morbilidad asociada a una traqueotomía. Por lo que mantenemos como técnica de elección esta técnica antes que una traqueotomía como tratamiento de soporte en pacientes con enfermedades oncológicas y parálisis bilateral de cuerdas vocales.

\section{CONCLUSIONES}

Según nuestra serie, la cordectomía posterior con láser $\mathrm{CO}_{2}$ obtiene unos buenos resultados en la resolución de la disnea en pacientes diagnosticados de parálisis bilateral de cuerdas vocales.

Se trata de un procedimiento relativamente sencillo y con una baja tasa de complicaciones.

En pacientes mayores debe tenerse en cuenta a la hora de informar preoperatoriamente que pueden presentar con mayor frecuencia una voz áfona mayor que el resto de la población de menor edad.

En este grupo de pacientes consideramos de especial relevancia que sean valorados en el postoperatorio inmediato de manera específica en una unidad de disfagia y aspiración ya que de manera más frecuente precisarán pautas para mejorar su función deglutoria en el corto y medio plazo.

\section{BIBLIOGRAFÍA}

1. Gupta J, Varshney S, Bist S, Bhagat S. Clinico-Etiolological Study of Vocal Cord Paralysis. Indian J Otolaryngol Head Neck Surg. 2013;65(1):16-9.

2. Sapundzhiev N, Lichtenberger G, Eckel $\mathrm{HE}$, Friedrich G, Zenev I, Toohill RJ, Werner JA. Surgery of adult bilateral vocal fold paralysis in adduction: history and trends. Eur Arch Otorhinolaryngol. 2008;265:1501-14. 
3. Young VN, Rosen CA. Arytenoid and posterior vocal fold surgery for bilateral vocal fold immobility. Curr Opin Otolaryngol Head Neck Surg. 2011, 19:422-7.

4. Paniello RC. Laryngeal reinnervation. Otolaryngol Clin North Am. 2004; 37: 161-81.

5. Li Y, Pearce EC, Mainthia R, Athavale SM, Dang J, Ashmead DH, et al. Comparison of ventilation and voice outcomes between unilateral laryngeal pacing and unilateral cordotomy for the treatment of bilateral vocal fold paralysis. ORL J Otorhinolaryngol Relat Spec. 2013;75:68-73.

6. Kojima A, Imoto Y, Osawa Y, Fujieda S. Predictor of rehabilitation outcome for dysphagia. Auris Nasus Larynx. 2014;41(3):294-8.

7. Mujica VR, Conklin J. When it's hard to swallow. What to look for in patients with dysphagia. Postgrad Med. 1999; 105(7):131-4, 141-2, 145.

8. Cuyás JM, Pérez D, Cuyás JM, Ramos A. Disfagia y aspiración en el anciano. En: Jaume G, Tomás M. Manejo de la disfagia y aspiración. Salvat; 2007:716.

9. Kashima HK. Bilateral vocal fold motion impairment: pathophysiology and management by transverse cordotomy. Ann Otol Rhinol Laryngol. 1991;100:717-21.

10. Hans S, Grevier-Buchanan L, Laccourreye O, Brasnu D. Aerodynamic and acoustic parameters in CO 2 laser posterior transverse cordotomy for bilateral vocal fold paralysis. Acta Otolaryngol. 2000;120:330-5

11. Motta $S$, Moscillo L, Imperiali M, Carra $P$, Motta G. CO2 Laser Treatment of Bilateral Vocal Cord Paralysis in Adduction. ORL J Otorhinolaryngol Relat Spec. 2003;65:35965.

12. Dursen G, Gokcan K. Aerodynamic, acoustic and functional results of posterior transverse laser cordotomy forbilateral abductor vocal cord paralysis. J Laryngol Otol. 2006;120:282-8.
13. Bajaj $Y$, Sethi N, Shayah A, Harris AT, Henshaw $P$, Coatesworth AP, et al. Vocal fold paralysis: role of bilateral transverse cordotomy. J Laryngol Otol. 2009;122:1348-51.

14. Landa M, Luqui I, Gómez J, Martínez Z. Cordectomía posterior. Nuestra experiencia. Acta Otorrinolaringol Esp. 2012;63(1):26-30.

15. Ozdemir S, Tuncer U, Tarkan O, Kara $\mathrm{K}$, Surmelioglu O. Carbon dioxide laser endoscopic posterior cordotomy technique for bilateral abductor vocal cord paralysis. A 15-year experience. JAMA Otolaryngol Head Neck Surg. 2013;139(4):401-4.

16. Dennis DP, Kashima H. Carbon dioxide laser posterior cordectomy for treatment of bilateral vocal cord paralysis. Ann Otol Rhinol Laryngol. 1989;98:930-4.

17. Laccourreye O, Paz Escovar MI, Gerhardt J, Hans S, Biacabe B, Brasnu D. CO2 laser endoscopic posterior partial transverse cordectomy for bilateral paralysis of the vocal fold. Laryngoscope. 1999;109:415-8.

18. Ossoff RH, Duncavage JA, Shapshay SM. Endoscopic laser arytenoidectomy revisited. Ann Otol Rhinol Laryngol. 1990;99:764-71.

19. Eckel HE, Thumfart M, Wassermann K, Vossing M, Thumfart WF. Cordectomy versus arytenoidectomy in the management of bilateral vocal cords paralysis. Ann Otol Rhinol Laryngol. 1994;103:852-7.

20. Yılmaz T, Süslü N, Atay $G$, Özer $S$, Günaydın RÖ, Bajin MD. Comparison of voice and swallowing parameters after endoscopic total and partial arytenoidectomy for bilateral abductor vocal fold paralysis. A randomized trial. JAMA Otolaryngol Head Neck Surg. 2013;139(7):712-8.

21. Pia F, Pisani P, Aluffi P. CO2 laser posterior ventriculocordectomy for the treatment of bilateral vocal cord paralysis. Eur Arch Otorhinolaryngol. 1999;256:403-6. 
22. Maurizi M, Paludetti G, Galli J, Cosenza A Di Girolamo S, Ottaviani F. Laser subtotal arytenoidectomy and posterior true and false cordotomy in the treatment of postthyroidectomy bilateral laryngeal fixation in adduction. Eur Arch Otorhinolaryngol. 1999; 256:291-5.

23. Mohamed NN, Sorour SS, El-Anwar MW, Quriba AS, Mahdy MA. Comparison between laser- and diathermy-assisted posterior cordotomy for bilateral vocal cord abductor paralysis. JAMA Otolaryngol Head Neck Surg. 2013;139(9):923-30.

24. Oysu C, Zer Toros S, Tepe-Karaca C, Şahin S, Sahin-Yilmaz A. Endoscopic posterior cordotomy with microdissection radiofrequency electrodes for bilateral vocal cord paralysis. Otolaryngol Head Neck Surgery. 2014;150:103-6.

25. Bosley B, Rosen CA, Blake Simpson C, McMullin BT, Gartner-Schmidt JL. Medial arytenoidectomy versus transverse cordotomy as treatment for bilateral vocal fold paralysis. Ann Otol Rhinol Laryngol. 2005;114(12):922-6.

26. Dispenza F, Dispenza C, Marchese D, Kulamarva G, Saraniti C. Treatment of bilateral vocal cord paralysis following permanent recurrent laryngeal nerve injury. Am J Otolaryngol 2012;33:285-8.

27. Harnisch W, Brosch S, Schmidt M, Hagen R. Breathing and voice quality after surgical treatment for bilateral vocal cord paralysis. Arch Otolaryngol Head Neck Surg. 2008;134:278-84.

28. Sataloff RT, Rosen DC, Hawkshaw M, Spiegel JR. The aging adult voice. J Voice. 1997;11:156-60.

29. Fernández S, Cobeta I, Vaca M. Presbifonía. En: Cobeta I, Núñez F, Fernández S. Patología de la voz. Ponencia oficial de la SEORL 2013. Marge Medica Books. 2013:305-12. 